OPEN ACCESS

Edited by:

Antonio Napolitano,

Bambino Gesù Children Hospital

(IRCCS), Italy

Reviewed by:

$S$ Senthil Kumaran,

All India Institute of Medical Sciences,

India

Kajari Bhattacharya,

Tata Memorial Hospital, India

${ }^{*}$ Correspondence:

Xin-Lan Xiao

jx_xiaoxinlan@sina.com

Specialty section: This article was submitted to

Cancer Imaging and

Image-directed Interventions,

a section of the journal

Frontiers in Oncology

Received: 24 March 2021

Accepted: 12 August 2021

Published: 02 September 2021

Citation:

Wang ZH, Xiao XL, Zhang ZT, He K and Hu F (2021) A Radiomics

Model for Predicting Early Recurrence in Grade // Gliomas

Based on Preoperative

Multiparametric Magnetic

Resonance Imaging

Front. Oncol. 11:684996.

doi: 10.3389/fonc.2021.684996

\section{A Radiomics Model for Predicting Early Recurrence in Grade II Gliomas Based on Preoperative Multiparametric Magnetic Resonance Imaging}

\author{
Zhen-hua Wang, Xin-Lan Xiao ", Zhao-Tao Zhang, Keng He and Feng Hu \\ Department of Radiology, The Second Affiliated Hospital of Nanchang University, Nanchang, China
}

Objective: This study aimed to develop a radiomics model to predict early recurrence $(<1$ year) in grade II glioma after the first resection.

Methods: The pathological, clinical, and magnetic resonance imaging (MRI) data of patients diagnosed with grade II glioma who underwent surgery and had a recurrence between 2017 and 2020 in our hospital were retrospectively analyzed. After a rigorous selection, 64 patients were eligible and enrolled in the study. Twenty-two cases had a pathologically confirmed recurrent glioma. The cases were randomly assigned using a ratio of 7:3 to either the training set or validation set. T1-weighted image (T1WI), T2weighted image (T2WI), and contrast-enhanced T1-weighted image (T1CE) were acquired. The minimum-redundancy-maximum-relevancy (mRMR) method alone or in combination with univariate logistic analysis were used to identify the most optimal predictive feature from the three image sequences. Multivariate logistic regression analysis was then used to develop a predictive model using the screened features. The performance of each model in both training and validation datasets was assessed using a receiver operating characteristic (ROC) curve, calibration curve, and decision curve analysis (DCA).

Results: A total of 396 radiomics features were initially extracted from each image sequence. After running the mRMR and univariate logistic analysis, nine predictive features were identified and used to build the multiparametric radiomics model. The model had a higher AUC when compared with the univariate models in both training and validation data sets with an AUC of 0.966 (95\% confidence interval: 0.949-0.99) and 0.930 (95\% confidence interval: 0.905-0.973), respectively. The calibration curves indicated a good agreement between the predictable and the actual probability of 
developing recurrence. The DCA demonstrated that the predictive value of the model improved when combining the three MRI sequences.

Conclusion: Our multiparametric radiomics model could be used as an efficient and accurate tool for predicting the recurrence of grade II glioma.

Keywords: radiomics, grade II gliomas, MRI, multiparametric, recurrence

\section{INTRODUCTION}

Glioma is a brain tumor originating from central glial cells with a high mortality rate (1-3). According to the World Health Organization (WHO), grade I and grade II tumors are classified as low-grade gliomas (LGG). LGGs are generally benign, with a recurrence rate of about $36 \%(4)$. Nevertheless, the clinical course of LGG may be unpredictable, as some of these tumors recur soon after primary treatment and/or undergo malignant transformation (5-7). A previous report indicated that low-grade gliomas (WHO II grade) have a 5-year survival rate of as high as $50 \%$ (8). Surgical resection followed by chemoradiation is the standard treatment option for gliomas. However, the risk and timing of recurrence following treatment in LGG are still difficult to predict accurately (9-12). Therefore, there is a need to identify accurate indicators for early detection and recurrence to provide timely, optimal treatment and improve survival.

Although histological analysis of surgical specimens is still considered the gold standard to grade gliomas, it may not always provide an accurate result (13) as the small sample obtained during the biopsy may not always reflect the grading heterogeneity within the entire tumor $(14,15)$. A substantial assessment would require the acquisition of samples from multiple regions within the tumor currently not widely accepted in clinical practice. Furthermore, a biopsy is an invasive procedure and also carries some risk. The acquisition of repeated biopsies is not always considered to be ethical as it may aggravate patient suffering.

The factors leading to poor OS post-surgery in LGG are still not well understood. Previous studies identified age, the extent of the tumor resection, and the expression of specific genes, including Ki-67 and the isocitrate dehydrogenase 1 (IDH1), as indicators for OS (16). Yet, to our knowledge, there is no accurate quantitative tool that could be used to predict at an early stage the risk of recurrence following the first tumor resection, highlighting the need to develop predictive models.

An alternative method that can be used to assess tumor recurrence post-surgery is magnetic resonance imaging (MRI). Previous studies have shown that radiomics could be used to quantitatively extract and assess numerous imaging features to effectively differentiate between high and lowgrade gliomas $(17,18)$ and differentiate tumor recurrence from radiation necrosis (19). When combined with clinical data, imaging features could be used to assess the OS and hence optimize the treatment for the patient. Therefore, this study aimed to create a radiomics model based on clinical and imaging features to predict the risk of developing recurrence in grade II glioma after the first resection.

\section{MATERIALS AND METHODS}

\section{Participants}

Retrospective analyses were performed on the follow-up medical records of 103 adult patients with histologically confirmed supratentorial grade II gliomas (according to WHO 2016 classification). All patients who had their first extensive glioma resection between May 2017 and November 2019 were included in the study. All patients had a MRI T1-contrast enhanced (T1CE) examination within $72 \mathrm{~h}$ after surgery to exclude the presence of a conspicuous residual tumor after surgery and received the same adjuvant chemoradiation treatment using a radiotherapy dose of $50.4 \mathrm{~Gy}$ in 28 fractions and $75 \mathrm{mg} / \mathrm{m}^{2}$ of temozolomide orally (20). Patients below 18 years with poor MRI images and tumor hemorrhage were excluded from the study (Figure 1). A total of 64 patients were ultimately included in the study.

\section{Data Collection}

After being discharged, the patients were regularly followed up by the neurosurgery group of the hospital. A periodical MRI examination was performed after treatment, and any tumor progression was noted in the patient's medical records according to the neuro-oncology (RANO) criteria (21). A biopsy was performed in those patients who had an obvious tumor progression noted on the MRI to further confirm the findings. The age, sex, progression-free survival (PFS), Ki-67, and IDH1 mutations were obtained from the patients' medical records. Three magnetic resonance imaging (MRI) sequences, including T1-weighted (T1W1), T2-weighted (T2WI), and T1contrast enhanced (T1CE), were acquired.

\section{MRI Parameters}

All the patients underwent multi-sequence imaging protocol on a 3.0 Tesla MRI system (Discovery 750; GE Healthcare, Milwaukee, WI, USA), with an eight-channel head coil (GE Healthcare, Chicago, IL, USA). For the T1-weighted image (T1WI) acquisition, the repetition time/echo time (TR/TE), matrix size, field-of-view (FOV), slice thickness, slice gap, and acquisition time were $1,750 / 25.4 \mathrm{~ms} ; 512 \times 512,220 \times 220,5 \mathrm{~mm}, 1.5 \mathrm{~mm}$, and $89 \mathrm{~s}$, respectively. For the T2WI acquisition, the (TR/TE), matrix size, FOV, layer thickness, layer spacing, and the number of layers were $4600 / 102 \mathrm{~ms}, 224 \times 320,220 \times 220,6 \mathrm{~mm}, 1$, and 18, respectively. 


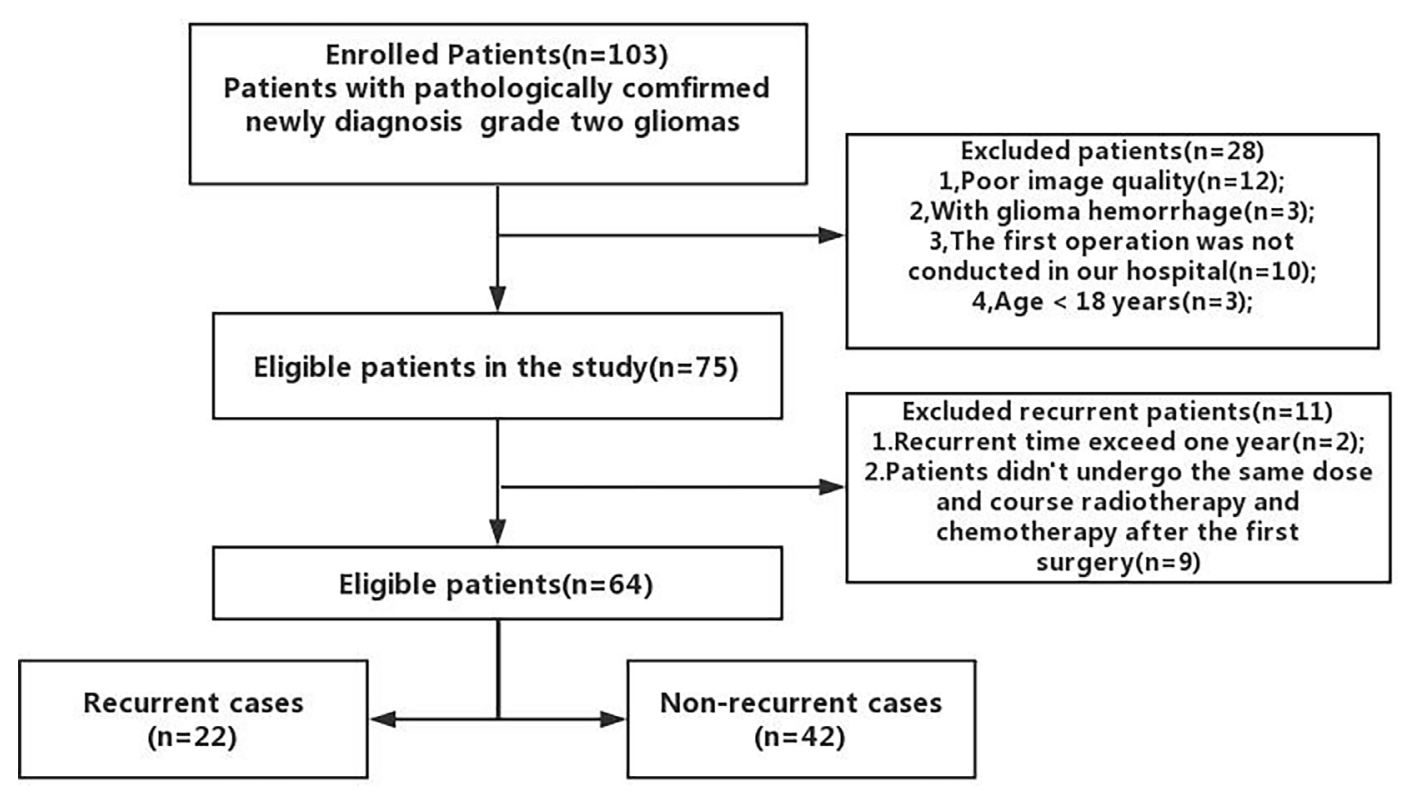

FIGURE 1 | Flow diagram illustrating the patient selection process.

The axial T1CE sequence was acquired by repeating the T1WI described above after a bolus injection of $0.1 \mathrm{mmol} / \mathrm{kg}$ of gadodiamide (Omniscan, GE Healthcare, Cork, Ireland).

\section{Description of the Region of Interest and Assessment of the MRI Sequences}

The ITK-snap software (www.itk-snap.org) was used to analyze the MRIs. A region of interest (ROI) was blindly delineated by two senior radiologists with more than 10 years of work experience. The boundaries of most low-grade tumors without contrast enhancement were determined on the T2WI images as these images are widely accepted in the identification of hyperintense signals representing the tumor regions (22). Then, the contours of the tumor delineated on the T2WI were transferred to the T1WI and T1CE images. In tumors with contrast enhancement, the tumor boundaries were delineated on the T1CE images by selecting the enhanced region. The delineated region was transferred onto the T1WI and T2WI images.

After the delineation of the ROI, all the patients were divided into the recurrent group (RG) and non-recurrent group (NRG) based on the RANO criteria (indicated in Table 1) and biopsy findings by two radiologists. In case of any disagreement, a consensus was reached through discussion, especially when there was a discrepancy between the two readers, as illustrated in Figure 2.

\section{Feature Extraction}

Radiomic features were extracted using the AK software (Artificial Intelligence Kit V3.0.0.R, GE Healthcare). A total of

TABLE 1 | RANO criteria used to evaluate treatment response in low-grade gliomas.

\begin{tabular}{|c|c|c|c|c|}
\hline Criterion & Complete remission & Partial remission & Stable disease & Progress disease \\
\hline T1CE & Not seen & Decrease $\geq 50 \%$ & $\begin{array}{l}\text { Increase or decrease in the range } \\
\text { of }-25 \% \sim+25 \%\end{array}$ & Increase $\geq 25 \% *$ \\
\hline T2WI/FLAIR & Stable or diminished & Stable or diminished & Stable or diminished & Increase ${ }^{\star}$ \\
\hline New lesion & $\begin{array}{l}\text { None (apart from those consistent } \\
\text { with radiation effects, and no new } \\
\text { or increased enhancement) }\end{array}$ & $\begin{array}{l}\text { None (apart from those consistent } \\
\text { with radiation effects, and no new } \\
\text { or increased enhancement) }\end{array}$ & $\begin{array}{l}\text { None (apart from those consistent } \\
\text { with radiation effects, and no new } \\
\text { or increased enhancement) }\end{array}$ & Present* \\
\hline Corticosteroids & None & Stable or diminished & Stable or diminished & Not apply \\
\hline Clinical status & Stable or improved & Stable or improved & Stable or improved & $\begin{array}{l}\text { Deteriorative* (not attributable to other } \\
\text { causes apart from the tumor, or } \\
\text { decrease in corticosteroid dose) }\end{array}$ \\
\hline $\begin{array}{l}\text { Requirement for } \\
\text { response }\end{array}$ & All & All & All & Any \\
\hline
\end{tabular}

CE, contrast-enhanced; FLAIR, fluid-attenuated inversion recovery.

*Progress is determined by anyone project. 


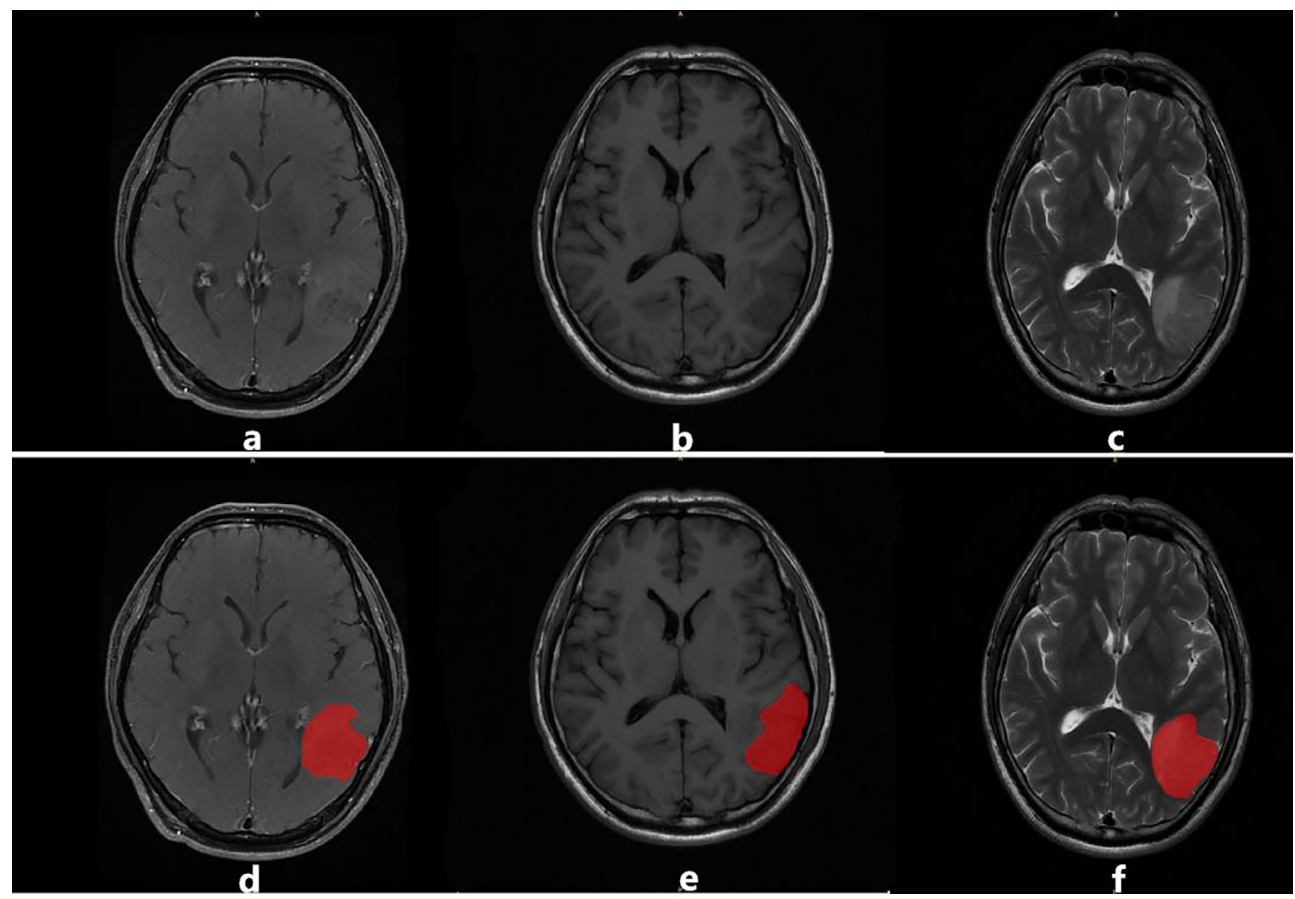

FIGURE 2 | An example of image segmentation: (A-C) illustrate T1CE, T1WI, and T2WI sequences, respectively. Images (D-F) illustrate the region of interest (ROI) in red delineated by the radiologists for feature extraction.

396 features were extracted from each MRI sequence, including the Laplacian of Gaussian (LoG), rotation invariant local binary patterns (RILBP), the gray level co-occurrence matrix (GLCM), intensity-based features (IBF), directional Gabor texture features (DGTF), and rotation invariant circular Gabor features (RICGF), etc. These features were then used to construct the multiparametric model.

\section{Data Preprocessing and Feature Screening}

The dataset was randomly categorized into the training or validation set using a ratio of 7:3. All cases in the training set were used to train the predictive model, while cases in the validation set were used to evaluate the model's performance independently. Variables with zero variance were excluded from the analysis. The missing values were substituted with the median value. Finally, the z-score was used to standardize the data (23). Feature screening was performed by using the minimum redundancy-maximum relevance (mRMR) (24) method alone or in combination with univariate logistic analysis. A $p$-value below 0.05 was deemed statistically significant.

\section{Development and Validation of Models}

Logistic regression analysis was used to construct predictive models based on the extracted optimal feature subsets of the training dataset. A receiver operator curve (ROC) was used to assess the performance of the radiomics models, and the sensitivity, specificity, and area under the curve (AUC) were calculated using five-fold cross validation. Calibration curves and decision curve analyses (DCA) were used to assess the clinical predictive performance of the models. The models were constructed using the $\mathrm{R}$ software (version 4.0.2), and a twotailed $p$-value below 0.05 was deemed statistically significant.

\section{Statistical Analysis}

According to the normality of samples based on the ShapiroWilk test, the independent samples t-test, the chi-square $\left(\mathrm{x}^{2}\right)$ test, Fisher's exact test and the Mann-Whitney U-test were used to identify any differences in age, gender, and other baseline characteristics between the training set and validation set. This data was analyzed using the statistical package for the social sciences (SPSS) version 22.0 software.

\section{Ethical Considerations}

Ethical approval was obtained from our hospital ethics committee. The need to obtain informed consent from patients was waived due to the retrospective nature of the study.

\section{RESULTS}

\section{Patient Characteristics}

The characteristics of the tumors and patients are summarized in Table 2. A total of 64 patients were included in the analysis. Following the first surgical resection, 64 patients were confirmed as grade II gliomas. According to the RANO criteria, 29 patients 
TABLE 2 | Baseline demographics and clinical characteristics of patients in the training and validation datasets.

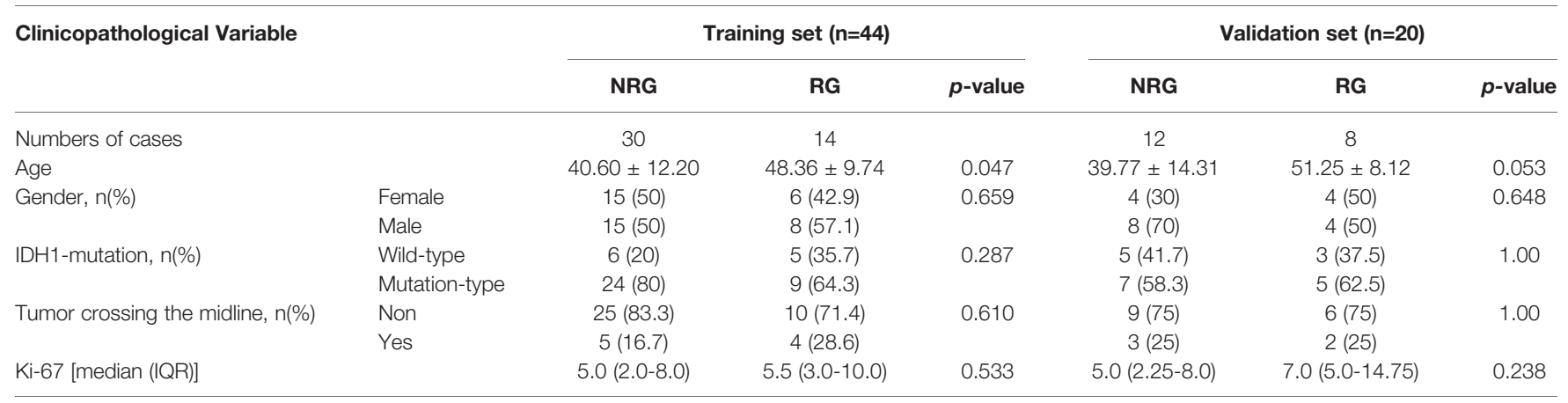

NRG, Non-recurrent group; $R G$, recurrent group; IQR, interquartile range.

were thought to have a tumor recurrence and underwent a biopsy. The biopsy confirmed the recurrence in 22 patients, while the other 7 patients were diagnosed with pseudo-response.

\section{Clinicopathological Characteristics}

Among the 64 patients included in the study, 22 had a pathologically confirmed recurrent tumor, and the rest did not have any recurrence. The patients were randomly divided into training and validation datasets using a ratio of 7:3. The baseline characteristics of the subjects are summarized in Table 2. There was no significant difference in the age $(p=0.251)$, gender $(p=$ $0.475)$, frequency of glioma recurrence $(p=0.845), \mathrm{Ki}-67(p=$ $0.486)$, and IDH1 $(p=0.885)$ mutation status and tumors crossing the midline $(p=0.307)$ between the training and validation group. There was a statistically significant difference $(p<0.05)$ in age between the RG and NRG in the training set. All other clinicopathological features did not differ significantly between the two groups.

\section{Performance of the Radiomics Models}

We extracted 396 features from the ROIs of every sequence. After running the mRMR algorithm, six features were selected from the T1WI images, five features from the T2WI images, and four features from the T1CE images. These three sequences were subsequently combined to identify the most important predictive features of the multiparametric model. Based on the univariate logistic analysis and mRMR, nine predictive features were eventually identified, and their correlation coefficients are illustrated in Figure 3. The low correlation coefficient between the nine features indicates little redundancy among every feature cluster.

The features screened from the T1WI, T2WI, T1CE, and multiparametric sequences are summarized in Table 3. Four radiomics models were established for predicting tumor recurrence based on the screened optimal predictive features and their contributing predictive weight for each image sequence, as illustrated in Table 3. In the T1WI sequence, six predictive features were included in the model, eventually resulting in an AUC of 0.842 and 0.79 in the training and validation datasets, respectively. In the T2WI sequence, five predictive features were used to construct the models, resulting in an AUC of 0.785 in the training set and 0.790 in the

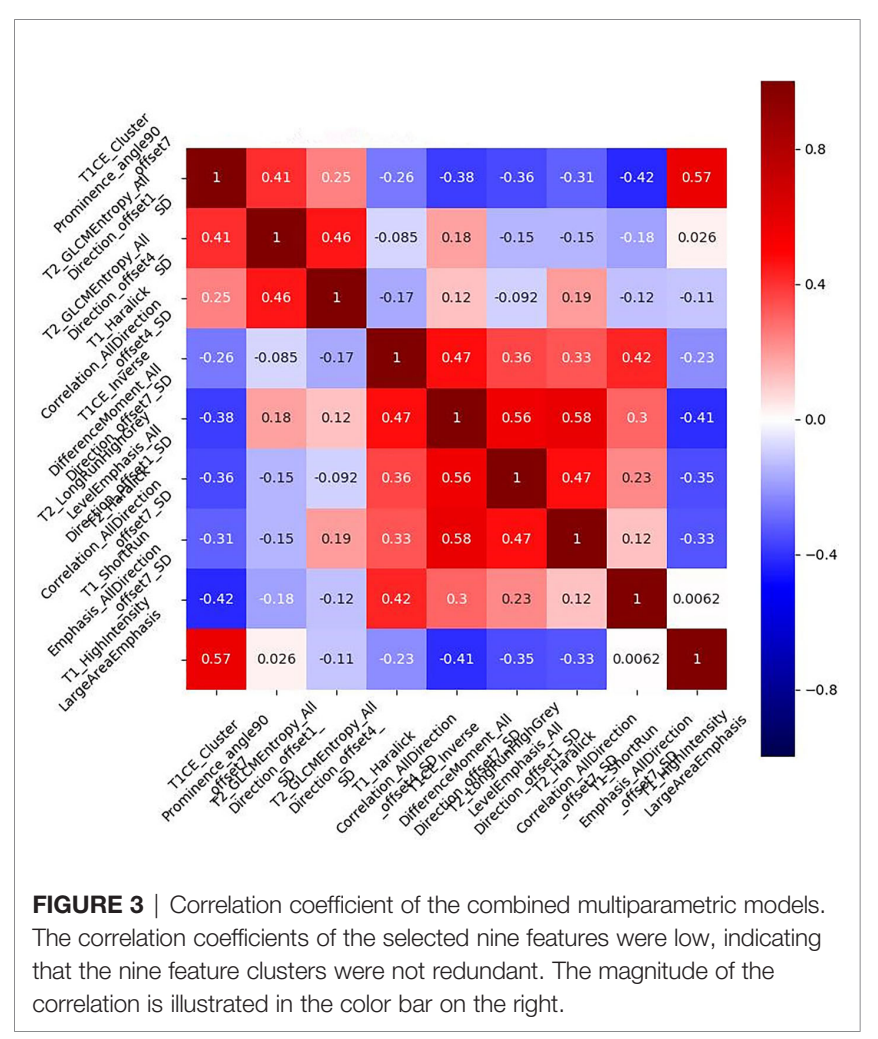

validation set. In the T1CE sequence, four predictive features were used to develop the predictive model, which resulted in an AUC of 0.784 in the training set and 0.803 in the validation set. The multiparametric MRI model included nine predictive features from the T1WI, T2WI, and T1CE sequence, resulting in the best overall performance with an AUC of 0.966 and 0.930 for the training and validation datasets, respectively (Table 4 and Figure 4). The calibration curves of the model also indicated a good agreement between the predicted probability and actual tumor recurrence both in the training set and validation set, indicating that the model was well-calibrated (Figure 5).

The DCA for the individual T1WI, T2WI, T1CE, and these combined multiparametric models are illustrated in Figure 6. The net benefit of the model constructed based on the three sequences was higher than the one based on the individual 
TABLE 3 | The screened features and their coefficients in the models for the different imaging sequences.

\begin{tabular}{|c|c|c|c|c|c|c|c|}
\hline Modality & Variables & Coefficient. & Std.Err. & $\mathbf{Z}$ & $P>|z|$ & {$[0.025$} & $0.975]$ \\
\hline \multirow[t]{5}{*}{ T1CE } & Intercept & -0.3825 & 0.5451 & -0.7017 & 0.4828 & -1.4508 & 0.6858 \\
\hline & GLCMEntropy_AllDirection_offset4_SD & 0.5540 & 0.5905 & 0.9382 & 0.3481 & -0.6034 & 1.7114 \\
\hline & Compactness2 & 0.6443 & 0.6101 & 1.0560 & 0.2910 & -0.5515 & 1.8401 \\
\hline & ShortRunEmphasis_AllDirection_offset7_SD & -1.1118 & 0.9869 & -1.1265 & 0.2600 & -3.0461 & 0.8226 \\
\hline & LongRunEmphasis_angle0_offset1 & -1.2584 & 0.7682 & -1.6382 & 0.1014 & -2.7640 & 0.2472 \\
\hline \multirow[t]{7}{*}{ T1WI } & intercept & 0.0196 & 0.3750 & 0.0523 & 0.9583 & -0.7154 & 0.7546 \\
\hline & GLCMEntropy_AllDirection_offset7_SD & 0.9189 & 1.1705 & 0.7850 & 0.4324 & -1.3753 & 3.2132 \\
\hline & LowGreyLeveIRunEmphasis_AllDirection_offset1_SD & -3.9480 & 3.5082 & -1.1254 & 0.2604 & -10.8240 & 2.9279 \\
\hline & RunLengthNonuniformity_AllDirection_offset4_SD & 0.3930 & 0.4920 & 0.7987 & 0.4245 & -0.5714 & 1.3573 \\
\hline & ShortRunEmphasis_AllDirection_offset7_SD & -0.3207 & 0.4130 & -0.7765 & 0.4375 & -1.1302 & 0.4888 \\
\hline & ShortRunEmphasis_angle90_offset4 & 1.0657 & 2.3549 & 0.4525 & 0.6509 & -3.5498 & 5.6812 \\
\hline & Variance & 1.7391 & 0.7825 & 2.2225 & 0.0262 & 0.2054 & 3.2728 \\
\hline \multirow[t]{6}{*}{ T2WI } & intercept & -0.1973 & 0.4298 & -0.4591 & 0.6461 & -1.0397 & 0.6451 \\
\hline & ClusterShade_angle45_offset7 & 0.0706 & 0.3401 & 0.2075 & 0.8356 & -0.5960 & 0.7372 \\
\hline & Correlation_AllDirection_offset1_SD & -0.4181 & 0.4751 & -0.8800 & 0.3788 & -1.3494 & 0.5131 \\
\hline & Sphericity & -0.6831 & 0.4032 & -1.6941 & 0.0902 & -1.4734 & 0.1072 \\
\hline & HighIntensityLargeAreaEmphasis & -0.0726 & 0.4370 & -0.1662 & 0.8680 & -0.9291 & 0.7838 \\
\hline & LongRunEmphasis_angle90_offset1 & -1.4411 & 0.9134 & -1.5777 & 0.1146 & -3.2313 & 0.3491 \\
\hline \multirow[t]{10}{*}{ Multiparametric } & intercept & -0.1338 & 2.3732 & -0.0564 & 0.9550 & -4.7852 & 4.5176 \\
\hline & T1CE_ClusterProminence_angle90_offset7 & -2.4287 & 2.7045 & -0.8980 & 0.3692 & -7.7295 & 2.8721 \\
\hline & T1CE_InverseDifferenceMoment_AllDirection_offset7_SD & 2.3638 & 3.4494 & 0.6853 & 0.4932 & -4.3970 & 9.1245 \\
\hline & T2_GLCMEntropy_AllDirection_offset1_SD & 2.0994 & 2.7421 & 0.7656 & 0.4439 & -3.2750 & 7.4739 \\
\hline & T2_GLCMEntropy_AllDirection_offset4_SD & -1.2254 & 0.0745 & 1.683 & 0.0924 & -0.8635 & 1.5517 \\
\hline & T2_LongRunHighGrayLevelEmphasis_AllDirection_offset1_SD & 0.9696 & 1.2682 & 0.7646 & 0.4445 & -1.5161 & 3.4553 \\
\hline & T2_HaralickCorrelation_AllDirection_offset7_SD & -10.1476 & 6.6254 & -1.5316 & 0.1256 & -23.1332 & 2.8380 \\
\hline & T1_HaralickCorrelation_AllDirection_offset4_SD & -1.8642 & 1.6618 & -1.1218 & 0.2620 & -5.1213 & 1.3929 \\
\hline & T1_ShortRunEmphasis_AllDirection_offset7_SD & -1.9502 & 1.4283 & -1.3654 & 0.1721 & -4.7497 & 0.8493 \\
\hline & T1_HighlntensityLargeAreaEmphasis & 2.6325 & 1.6886 & 1.5590 & 0.1190 & -0.6770 & 5.9420 \\
\hline
\end{tabular}

TABLE 4 | The performance of the models for predicting tumor recurrence in the training and validation datasets.

\begin{tabular}{|c|c|c|c|c|c|c|c|}
\hline Modality & $\begin{array}{c}\text { Features screening } \\
\text { method }\end{array}$ & $\begin{array}{l}\text { Number of features } \\
\text { after screened }\end{array}$ & Cohort & AUC (95\% Cl) & Sensitivity & Specificity & Accuracy \\
\hline \multirow[t]{2}{*}{ T1WI } & mRMR & 6 & Training & $0.842(0.674-0.905)$ & 0.7 & 0.7 & 0.75 \\
\hline & & & Validation & $0.79(0.687-0.902)$ & 0.778 & 0.778 & 0.78 \\
\hline \multirow[t]{2}{*}{ T2WI } & mRMR & 5 & Training & $0.785(0.697-0.912)$ & 0.727 & 0.682 & 0.705 \\
\hline & & & Validation & 0.79 (0.679-0.92) & 0.8 & 0.5 & 0.65 \\
\hline \multirow[t]{2}{*}{ T1CE } & $\mathrm{mRMR}$ & 4 & Training & $0.784(0.665-0.913)$ & 0.889 & 0.556 & 0.722 \\
\hline & & & Validation & $0.802(0.693-0.911)$ & 0.78 & 0.778 & 0.8 \\
\hline \multirow[t]{2}{*}{ Multi-modalities } & ULA + mRMR & 9 & Training & 0.966 (0.949-0.99) & 0.905 & 0.952 & 0.929 \\
\hline & & & Validation & 0.93 (0.905-0.973) & 1 & 0.8 & 0.90 \\
\hline
\end{tabular}

ULA, univariate logistic analysis.

imaging sequence, to which it was superior across nearly the entire range of clinically useful threshold risks.

\section{DISCUSSION}

Surgery followed by chemoradiation is the main treatment option for patients diagnosed with LGG. Tumor recurrence post-treatment is one of the factors leading to poor OS. Surgical resection is one of the treatment options for patients diagnosed with recurrent LGG. Still, guidelines issued by several professional bodies state that there is limited high-level clinical evidence on the effectiveness of a secondary invasive resection on survival. A study by Patrizz et al. (25) indicated that histopathologic findings following chemoradiation do not always correlate with clinical outcomes in patients diagnosed with recurrence post-surgery. First of all, the pathological specimens may not always reflect the nature of the whole tumor. Furthermore, several studies found that other clinical factors that may have an impact on survival including age, radiotherapy dose, and the extent of tumor resection (16). Multiparametric MRI has played an important role in distinguishing between LGG and HGG as well as recurrence from radiation-induced necrosis. However, to our knowledge, currently, there is no suitable clinical and image-based predictive model to assess the risk of recurrence post-surgery in LGG patients. Therefore in this study, we made use of the imaging data of 64 LGG patients to develop a model that could be used to predict recurrence in these patients and hence enable clinicians to identify the patients that are most likely to benefit from additional surgery.

In our study, there was no difference in the baseline characteristics between the RG and NRG except for age. Consistent with the retrospective study by $\mathrm{Li}$ et al. (16), age 


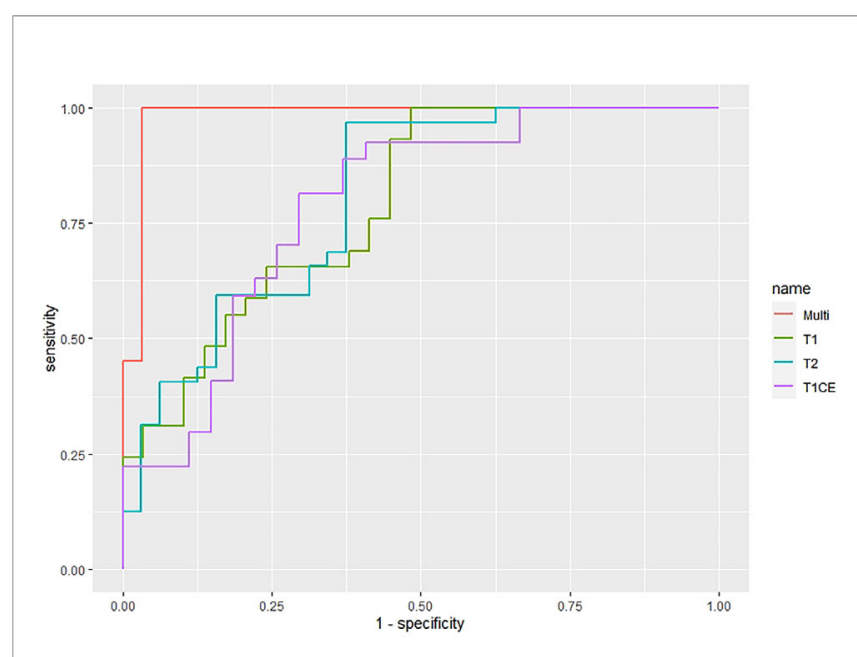

FIGURE 4 | The ROC curves of the four imaging prediction models whereby the green curve represents the T1WI model, the blue curve represents the $\mathrm{T} 2 \mathrm{WI}$, the purple curve represents T1CE, and the red curve represents the multiparametric MRI model.

was found to be an important risk factor for recurrence in grade II gliomas following the first surgery. Jansen et al. (26) conducted a long-term follow-up of 110 patients with LGG (WHO Grade II) after resection. Their results demonstrated that the initial extent of the resection influenced the progression-free survival, time to malignant transformation, and overall survival. Moreover, Patrizz et al. (25) indicated that the radiotherapy dose after surgery has a significant impact on survival in LGG patients. In our study, all patients had an extensive tumor resection and received the same radiation dose. Therefore, the effects of these variables on tumor recurrence could not be assessed.

Studies have shown a high correlation between certain genetic alterations, recurrence, and prognosis in grade II and III gliomas. Mutations of the isocitrate dehydrogenase (IDH)1/2 genes are common events in gliomas (27), especially among grade II gliomas, where IDH1 mutations are observed in about $70 \%$ to $80 \%$ of cases $(27,28)$. Some studies indicated that IDH1 mutation status could improve OS and PFS in grade II and III glioma $(19,29)$. Although the IDH1 mutation has been identified as an independent positive prognostic biomarker for survival in patients with glioma $(26,30)$, the association between the IDH mutant status and the risk of developing recurrence is still not clear. In the present study, the proportion of IDH mutation cases was noticeably higher in NRG compared with RG [31/42(73.8\%) vs 14/22(63.6\%)]; however, the statistic results showed that there was not a significant difference between NRG and RG (Table 2), which indicated that there might not be a link between the IDHI mutation and tumor recurrence; nevertheless, due to the limitation of our relatively small sample size, it still needs a big sample for further verification.

The RANO criteria are still widely used to assess the tumor response post-treatment and the need for additional treatment $(31,32)$. Despite being used extensively, the accuracy rate of the RANO criteria in distinguishing between tumor recurrence and

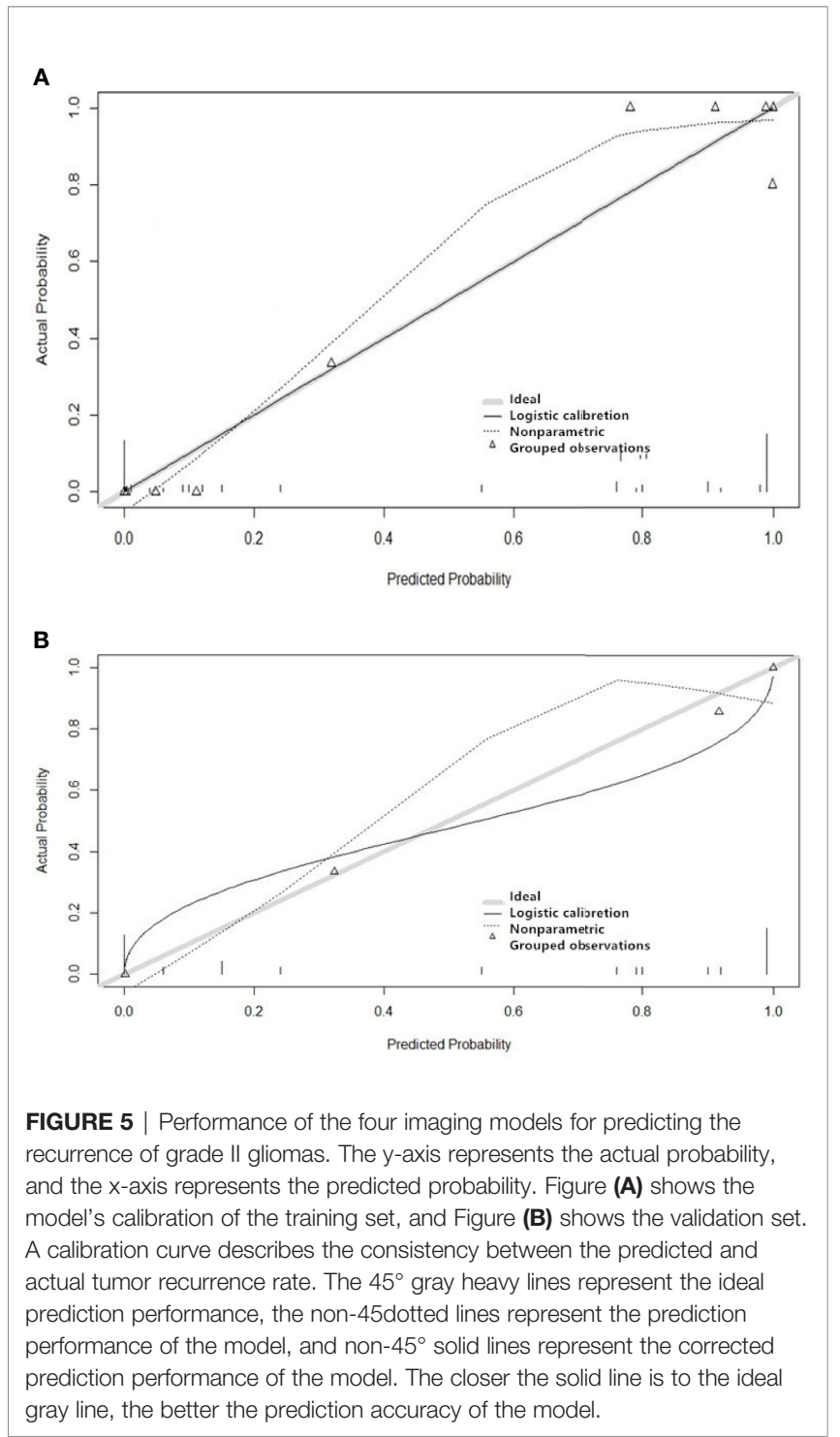

pseudo-response $(32,33)$ in our study was only $75.86 \%$. The multi-parameters radiomics model developed in our study resulted in higher prediction accuracy in both testing and validation datasets.

In order to develop our radiomics model, numerous features were extracted from each of the three MRI sequences. It is important to acknowledge that the sample size in our study was relatively small, potentially over-fitting the model (34). In order to reduce this risk, $\mathrm{mRMR}$ was used for feature dimensionality reduction. This technique has been widely used in several studies and involves selecting features from the mutually correlated distance or similarity score hence facilitating the data screening process $(35,36)$.

Numerous studies evaluated the use of radiomics models in predicting recurrence in glioma after radiotherapy. Wang et al. (37) proposed a radiomics model based on MRI and PET images to discriminate between tumor recurrence from radiation necrosis. The model performed well in both training and validation datasets with an AUC of 0.988 and 0.914 , 


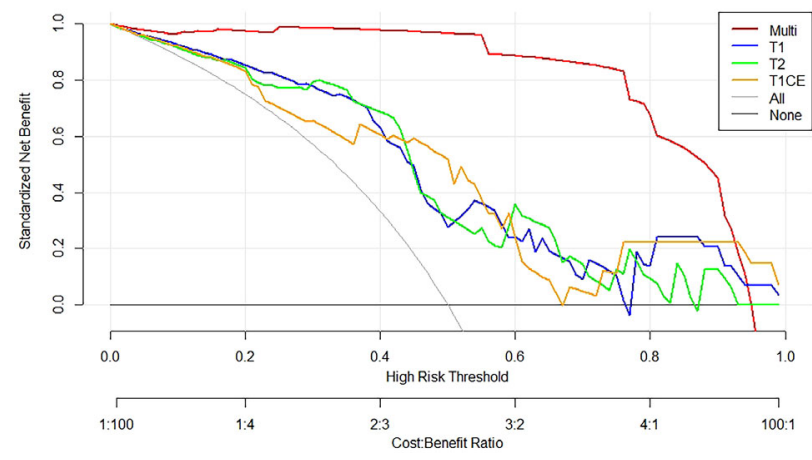

FIGURE 6 | Decision curve analysis constructed using the radiomics features extracted from T1WI, T2Wl, and T1CE sequences. The $y$-axis measures the net benefit of the T1WI (blue curve), T2WI (green curve), T1CE (yellow curve), and the multiparametric (red curve) images. The gray curve represents the assumption that all patients were treated, and the straight black line at the bottom of the figure represents the assumption that none of the patients were treated.

respectively. A similar model based on 51 glioma patients developed by Quan Zhang et al. (19) achieved outstanding performance with an AUC of 0.962 following validation. However, to the best of our knowledge, this is the first multiparametric model developed to predict recurrence in LGG before surgery. Our model also achieved an excellent performance, with an AUC of 0.966 and 0.930 in the testing and validation dataset, respectively.

In the study, a total of nine optimal features were selected for the construction of the multiparametric radiomics model. Among these features, there were three gray level run length matrix (GLRLM) features (T2_LongRunHighGrayLevel Emphasis_AllDirection_offset1_SD, T1_ShortRunEmphasis_ AllDirection_offset7_SD, and T1_ShortRunEmphasis_ AllDirection_offset7_SD), one gray level size zone matrix (GLSZM) feature (T1_HighIntensityLargeAreaEmphasis), and the rest were gray level co-occurrence matrix (GLCM) features (Table 3). The above results indicate that GLCM features played the most important role in the model. In some previous radiomics studies, the GLCM features also played an important role in predicting the IDH mutation status. Checkout et al. developed a new approach to predict IDH mutation status that outperformed competing methods (38), while Park et al. (39) found that GLCM was one of the strongest IDH status prediction factors. Furthermore, in a study by Chaddad et al. (40), GLCM had a significant role in predicting survival in patients with glioblastoma. Combined with these previous studies, we can reasonably infer that GLCM may convey information that could potentially be used to predict recurrence.

Both calibration and discrimination are valuable aspects of a prediction model (41). AUC is a common evaluation index of discrimination, while calibration reflects the level of agreement between the actual observed outcomes and the model's predicted outcomes (42). However, the AUC focuses merely on the predictive accuracy of the signature. As such, it does not tell us whether the model is worth using at all. DCA is a statistical method that incorporate consequences and, thus, can inform the decision of whether to use this model (43). Therefore to further complement the AUC findings, a DCA was also performed to evaluate the clinical value of the models (44). In our study, both the AUC and calibration curve (Figure 5) showed that our model has a high prediction accuracy. Furthermore, the DCA curves showed that within a relatively large threshold range, our proposed radiomics models could be used to improve the treatment decision-making process. However, the DCA showed that multiparametric MRI models had a significantly higher performance when compared with models based on a single MRI sequence across nearly the entire range of clinically useful threshold risks (Figure 6).

This study has some limitations that have to be acknowledged. The majority of the patients with recurrent LGG at our institution generally prefer to be treated with radiotherapy and chemotherapy as opposed to surgery. This limited the sample size in our study and hence limited the number of clinical, pathological, molecular, and imaging features that could be used to train the model. In order to improve the robustness and generalizability of the model, further studies with a larger sample from multiple institutions with a longer follow-up are warranted. A larger sample will also allow us to apply different machine learning strategies to improve the prediction performance of the model. Further research is also recommended to illustrate the relationship between specific imaging features and pathology. Finally, additional studies are also recommended to evaluate the impact of early recurrence prediction on the provision of timely interventions and ultimately survival.

\section{CONCLUSION}

The application of our radiomics model-based features extracted from multiparametric MRI could be used to predict the risk of early recurrence of grade II gliomas after the first surgical resection. This model could be used to guide the clinicians' decision on the need for further invasive treatment such as biopsy and surgery in LGG patients.

\section{DATA AVAILABILITY STATEMENT}

The raw data supporting the conclusions of this article will be made available by the authors, without undue reservation.

\section{ETHICS STATEMENT}

The studies involving human participants were reviewed and approved by The Second Affiliated Hospital of Nanchang University Medical Research Ethical Committee. Written informed consent for participation was not required for this study in accordance with the national legislation and the institutional requirements. 


\section{AUTHOR CONTRIBUTIONS}

Conceptualization: Z-hW, X-LX, and KH. Data curation: Z-hW. Formal analysis: Z-hW, X-LX, and KH. Funding acquisition: Z-hW and X-LX. Investigation: Z-hW, X-LX, and FH. Methodology: Z-hW, X-LX, and Z-TZ. Project administration: Z-hW, X-LX, and KH. Resources: Z-hW, X-LX, and KH. Software: Z-hW, X-LX, and FH. Supervision: Z-hW, X-LX, and KH. Validation: Z-hW, X-LX, and Z-TZ. Visualization: Z-hW, $\mathrm{X}-\mathrm{LX}$, and Z-TZ. Writing-original draft: Z-hW and X-LX. Writing-review and editing: Z-hW and X-LX.

\section{REFERENCES}

1. Ganau L, Paris M, Ligarotti G, Ganau M. Management of Gliomas: Overview of the Latest Technological Advancements and Related Behavioral Drawbacks. Behav Neurol (2015) 2015:862634. doi: 10.1155/2015/862634

2. Tully PA, Gogos AJ, Love C, Liew D, Drummond KJ, Morokoff AP. Reoperation for Recurrent Glioblastoma and Its Association With Survival Benefit. Neurosurgery (2016) 79:678-89. doi: 10.1227/NEU.0000000000001338

3. Ostrom Q, Gittleman H, Fulop J, Liu M, Blanda R, Kromer C, et al. CBTRUS Statistical Report: Primary Brain and Central Nervous System Tumors Diagnosed in the United States in 2008-2012. Neuro Oncol (2015) 17(Suppl 4):iv1-iv62. doi: 10.1093/neuonc/nov189

4. Fukuya Y, Ikuta S, Maruyama T, Nitta M, Saito T, Tsuzuki S, et al. Tumor Recurrence Patterns After Surgical Resection of Intracranial Low-Grade Gliomas. J Neurooncol (2019) 144:519-28. doi: 10.1007/s11060-019-03250-8

5. Daniels TB, Brown PD, Felten SJ, Wu W, Buckner JC, Arusell RM, et al. Validation of EORTC Prognostic Factors for Adults With Low-Grade Glioma: A Report Using Intergroup 86-72-51. Int J Radiat Oncol Biol Phys (2011) 81:218-24. doi: 10.1016/j.ijrobp.2010.05.003

6. Okita Y, Narita Y, Miyakita Y, Ohno M, Matsushita Y, Fukushima S, et al. IDH1/2 Mutation Is a Prognostic Marker for Survival and Predicts Response to Chemotherapy for Grade II Gliomas Concomitantly Treated With Radiation Therapy. Int J Oncol (2012) 41:1325-36. doi: 10.3892/ijo.2012.1564

7. Komori T, Muragaki Y, Chernov MF. Pathology and Genetics of Gliomas. Prog Neurol Surg (2018) 31:1-37. doi: 10.1159/000466835

8. Goodenberger ML, Jenkins RB. Genetics of Adult Glioma. Cancer Genet (2012) 205(12):613-21. doi: 10.1016/j.cancergen.2012.10.009

9. Westphal M, Giese A. Local Control of Gliomas: The Next Best Step-A Good Step? Front Radiat Ther Oncol (1999) 33:214-26. doi: 10.1159/000061237

10. De Fazio S, Russo E, Ammendola M, Donato Di Paola E, De Sarro G. Efficacy and Safety of Bevacizumab in Glioblastomas. Curr Med Chem (2012) 19:97281. doi: 10.2174/092986712799320646

11. Tugcu B, Postalci L, Gunaldi O, Tanriverdi O, Akdemir H. Efficacy of Clinical Prognostic Factors on Survival in Patients With Glioblastoma. Turk Neurosurg (2010) 20:117-25. doi: 10.5137/1019-5149.JTN.2461-09.4

12. Thompson G, Lawrie TA, Kernohan A, Jenkinson MD. Interval Brain Imaging for Adults With Cerebral Glioma. Cochrane Database Syst Rev (2019) 12(12):CD013137. doi: 10.1002/14651858.CD013137.pub2

13. Kessler J, Hohmann T, Güttler A, Petrenko M, Ostheimer C, Hohmann U, et al. Radiosensitization and a Less Aggressive Phenotype of Human Malignant Glioma Cells Expressing Isocitrate Dehydrogenase 1 (IDH1) Mutant Protein: Dissecting the Mechanisms. Cancers (2019) 11(6):889. doi: 10.3390/cancers11060889

14. Kani Y, Cecere TE, Lahmers K, LeRoith T, Zimmerman KL, Isom S, et al. Diagnostic Accuracy of Stereotactic Brain Biopsy for Intracranial Neoplasia in Dogs: Comparison of Biopsy, Surgical Resection, and Necropsy Specimens. $J$ Vet Intern Med (2019) 33:1384-91. doi: 10.1111/jvim.15500

15. Reardon DA, Wen PY. Glioma in 2014: Unravelling Tumour Heterogeneityimplications for Therapy. Nat Rev Clin Oncol (2015) 12:6970. doi: 10.1038/nrclinonc.2014.223

16. Li J, Niu X, Gan Y, Yang Y, Wang T, Zhang H, et al. Clinical and Pathologic Features and Prognostic Factors for Recurrent Gliomas. World Neurosurg (2019) 128:e21-30. doi: 10.1016/j.wneu.2019.02.210

\section{FUNDING}

This work was supported and funded by the Key research and development projects in Jiangxi Province, China (NO.20171ACG70002).

\section{ACKNOWLEDGMENTS}

We acknowledge the Department of Radiology of The Second Affiliated Hospital of Nanchang University.

17. Tian Q, Yan LF, Zhang X, Zhang X, Hu YC, Han Y, et al. Radiomics Strategy for Glioma Grading Using Texture Features From Multiparametric MRI. J Magn Reson Imaging (2018) 48(6):1518-28. doi: 10.1002/jmri.26010

18. Wang Q, Li Q, Mi R, Ye H, Zhang H, Chen B, et al. Radiomics Nomogram Building From Multiparametric MRI to Predict Grade in Patients With Glioma: A Cohort Study. J Magn Reson Imaging: JMRI (2019) 49(3):825-33. doi: 10.1002/jmri.26265

19. Zhang Q, Cao J, Zhang J, Bu J, Yu Y, Tan Y, et al. Differentiation of Recurrence From Radiation Necrosis in Gliomas Based on the Radiomics of Combinational Features and Multimodality MRI Images. Comput Math Methods Med (2019) 2019:2893043. doi: 10.1155/2019/2893043

20. Shaw E, Arusell R, Scheithauer B, O'Fallon J, O'Neill B, Dinapoli R, et al. Prospective Randomized Trial of Low-Versus High-Dose Radiation Therapy in Adults With Supratentorial Low-Grade Glioma: Initial Report of a North Central Cancer Treatment Group/Radiation Therapy Oncology Group/ Eastern Cooperative Oncology Group Study. J Clin Oncol (2002) 20 (9):2267-76. doi: 10.1200/JCO.2002.09.126

21. Yamanaka T, Shirota S, Sawai Y, Murai T, Fujita N, Hosoi H. Osteoporosis as a Risk Factor for the Recurrence of Benign Paroxysmal Positional Vertigo. Laryngoscope (2013) 123(11):2813-6. doi: 10.1002/lary.24099

22. Li Y, Liu X, Xu K, Qian Z, Wang K, Fan X, et al. MRI Features can Predict EGFR Expression in Lower Grade Gliomas: A Voxel-Based Radiomic Analysis. Eur Radiol (2018) 28:356-62. doi: 10.1007/s00330-017-4964-Z

23. Kranzusch R, Aus dem Siepen F, Wiesemann S, Zange L, Jeuthe S, Ferreira da Silva $\mathrm{T}$, et al. Z-Score Mapping for Standardized Analysis and Reporting of Cardiovascular Magnetic Resonance Modified Look-Locker Inversion Recovery (MOLLI) T1 Data: Normal Behavior and Validation in Patients With Amyloidosis. J Cardiovasc Magn Reson (2020) 22(1):6. doi: 10.1186/s12968-019-0595-7

24. Peng H, Long F, Ding C. Feature Selection Based on Mutual Information: Criteria of Max-Dependency, Max-Relevance, and Min-Redundancy. IEEE Trans Pattern Anal Mach Intell (2005) 27(8):1226-38. doi: 10.1109/ TPAMI.2005.159

25. Patrizz A, Dono A, Zhu P, Tandon N, Ballester LY, Esquenazi Y. Tumor Recurrence or Treatment-Related Changes Following Chemoradiation in Patients With Glioblastoma: Does Pathology Predict Outcomes? J NeuroOncol (2021) 152(1):163-72. doi: 10.1007/s11060-020-03690-7

26. Jansen E, Hamisch C, Ruess D, Heiland DH, Goldbrunner R, Ruge M, et al. Observation After Surgery for Low Grade Glioma:Long-Term Outcome in the Light of the 2016 WHO Classification. J Neurooncol (2019) 145(3):501-7. doi: 10.1007/s11060-019-03316-7

27. Yan H, Parsons DW, Jin G, McLendon R, Rasheed BA, Yuan W, et al. IDH1 and IDH2 Mutations in Gliomas. N Engl J Med (2009) 360:765-73. doi: 10.1056/NEJMoa0808710

28. Balss J, Meyer J, Mueller W, Korshunov A, Hartmann C, von Deimling A. Analysis of the IDH1 Codon 132 Mutation in Brain Tumors. Acta Neuropathol (2008) 116:597-602. doi: 10.1007/s00401-008-0455-2

29. Jiang H, Cui Y, Wang J, Lin S. Impact of Epidemiological Characteristics of Supratentorial Gliomas in Adults Brought About by the 2016 World Health Organization Classification of Tumors of the Central Nervous System. Oncotarget (2017) 8(12):20354-61. doi: 10.18632/oncotarget.13555

30. Nobusawa S, Watanabe T, Kleihues P, Ohgaki H. IDH1 Mutations as Molecular Signature and Predictive Factor of Secondary Glioblastomas. Clin Cancer Res (2009) 15:6002-7. doi: 10.1158/1078-0432.CCR-09-0715 
31. Li T, Chen L, Gan M. Quality Control of Imbalanced Mass Spectra From Isotopic Labeling Experiments. BMC Bioinf (2019) 20(1):549. doi: 10.1186/ s12859-019-3170-1

32. Wick W, Chinot OL, Bendszus M, Mason W, Henriksson R, Saran F, et al. Evaluation of Pseudoprogression Rates and Tumor Progression Patterns in a Phase III Trial of Bevacizumab Plus Radiotherapy/Temozolomide for Newly Diagnosed Glioblastoma. Neuro Oncol (2016) 18(10):1434-41. doi: 10.1093/neuonc/now091

33. Ellingson BM, Wen PY, Cloughesy TF. Modified Criteria for Radiographic Response Assessment in Glioblastoma Clinical Trials. Neurotherapeutics (2017) 14(2):307-20. doi: 10.1007/s13311-016-0507-6

34. Balagurunathan Y, Kumar V, Gu Y, Kim J, Wang H, Liu Y, et al. Test-Retest Reproducibility Analysis of Lung CT Image Features. J Digit Imaging (2014) 27:805-23. doi: 10.1007/s10278-014-9716-x

35. Cai Y, Huang T, Hu L, Shi X, Xie L, Li Y. Prediction of Lysine Ubiquitination With mRMR Feature Selection and Analysis. Amino Acids (2012) 42(4):138795. doi: 10.1007/s00726-011-0835-0

36. Alshamlan H, Badr G, Alohali Y. mRMR-ABC: A Hybrid Gene Selection Algorithm for Cancer Classification Using Micro-Array Gene Expression Profiling. BioMed Res Int (2015) 2015:604910. doi: 10.1155/2015/604910

37. Wang K, Qiao Z, Zhao X, Li X, Wang X, Wu T, et al. Individualized Discrimination of Tumor Recurrence From Radiation Necrosis in Glioma Patients Using an Integrated Radiomics-Based Model. Eur J Nucl Med Mol Imaging (2020) 47(6):1400-11. doi: 10.1007/s00259-019-04604-0

38. Chekouo T, Mohammed S, Rao A. A Bayesian 2D Functional Linear Model for Gray-Level Co-Occurrence Matrices in Texture Analysis of Lower Grade Gliomas. NeuroImage Clin (2020) 28:102437. doi: 10.1016/j.nicl.2020.102437

39. Park CJ, Choi YS, Park YW, Ahn SS, Kang SG, Chang JH, et al. Diffusion Tensor Imaging Radiomics in Lower-Grade Glioma: Improving Subtyping of Isocitrate Dehydrogenase Mutation Status. Neuroradiology (2020) 62(3):31926. doi: 10.1007/s00234-019-02312-y

40. Chaddad A, Daniel P, Desrosiers C, Toews M, Abdulkarim B. Novel Radiomic Features Based on Joint Intensity Matrices for Predicting Glioblastoma Patient
Survival Time. IEEE J BioMed Health Inform (2019) 23:795-804. doi: 10.1109/ JBHI.2018.2825027

41. Lagendijk J, Steyerberg EW, Daalderop LA, Been JV, Steegers E, Posthumus AG. Validation of a Prognostic Model for Adverse Perinatal Health Outcomes. Sci Rep (2020) 10(1):11243. doi: 10.1038/s41598-020-68101-3

42. Gerry S, Bonnici T, Birks J, Kirtley S, Virdee PS, Watkinson PJ, et al. Early Warning Scores for Detecting Deterioration in Adult Hospital Patients: Systematic Review and Critical Appraisal of Methodology. BMJ (Clin Res Ed.) (2020) 369:m1501. doi: 10.1136/bmj.m1501

43. Van Calster B, Wynants L, Verbeek J, Verbakel JY, Christodoulou E, Vickers AJ, et al. Reporting and Interpreting Decision Curve Analysis: A Guide for Investigators. Eur Urol (2018) 74:796-804. doi: 10.1016/j.eururo.2018.08.038

44. Vickers AJ, Elkin EB. Decision Curve Analysis: A Novel Method for Evaluating Prediction Models. Med Decision Making: An Int J Soc Med Decision Making (2006) 26(6):565-74. doi: 10.1177/0272989X06295361

Conflict of Interest: The authors declare that the research was conducted in the absence of any commercial or financial relationships that could be construed as a potential conflict of interest.

Publisher's Note: All claims expressed in this article are solely those of the authors and do not necessarily represent those of their affiliated organizations, or those of the publisher, the editors and the reviewers. Any product that may be evaluated in this article, or claim that may be made by its manufacturer, is not guaranteed or endorsed by the publisher.

Copyright (c) 2021 Wang, Xiao, Zhang, He and Hu. This is an open-access article distributed under the terms of the Creative Commons Attribution License (CC BY). The use, distribution or reproduction in other forums is permitted, provided the original author(s) and the copyright owner(s) are credited and that the original publication in this journal is cited, in accordance with accepted academic practice. No use, distribution or reproduction is permitted which does not comply with these terms. 